\title{
Automated Quantification of Regional Myocardial Perfusion by Analysis of Contrast-Enhanced Echocardiographic Images
}

\author{
EG Caiani ${ }^{1,2}$, CE Korcarz ${ }^{2}$, KA Collins ${ }^{2}$, S Cerutti ${ }^{1}$, RM Lang $^{2}$, V Mor-Avi ${ }^{2}$ \\ ${ }^{1}$ Dipartimento di Bioingegneria, Politecnico di Milano, Milano, Italy \\ ${ }^{2}$ Noninvasive Cardiac Imaging Laboratory, University of Chicago, Chicago, Illinois, USA
}

\begin{abstract}
Quantitative analysis of myocardial perfusion images is currently done by manual techniques. We hypothesized that the seeded region growing procedure, applied to power modulation images, would allow the frame-byframe detection of myocardial contours, thus eliminating the need for image realignment. We tested this approach in 13 anaesthetized pigs during continuous iv infusion of Definity (DuPont) at baseline, during LAD occlusion and reperfusion. Regional mean pixel intensity was calculated for each consecutive end-systolic frame, and fitted with $y=A \cdot\left(1-e^{-\beta \cdot t}\right)$. Perfusion defects caused by LAD occlusion were confirmed by a $44 \pm 27 \%$ decrease in $A$ and $a$ $42 \pm 25 \%$ decrease in $A \cdot \beta(p<0.05)$ in the LAD territory, while no changes were observed in non-ischemic segments. With reperfusion, $A, \boldsymbol{\beta}$ and $A \cdot \boldsymbol{\beta}$ increased above baseline levels $(p<0.05)$. This technique allows automated quantification of regional myocardial perfusion, without the need for manual tracing and realignment of regions of interest.
\end{abstract}

\section{Introduction}

Quantitative assessment of myocardial perfusion is currently based on subjective and time-consuming manual tracing and frame-by-frame realignment of myocardial regions of interest (ROIs). Recently, power modulation imaging was shown to provide homogeneous contrast enhancement in the left ventricular (LV) cavity, which allows accurate automated endocardial border detection [1,2]. We hypothesized that endocardial boundaries obtained from contrast-enhanced power modulation images could be used as a basis for frame-byframe identification of myocardial ROIs (Fig. 1). Accordingly, the goal of this study was to test the feasibility of automated myocardial segmentation and quantitative, translation-free analysis of tissue perfusion in an animal model of acute ischemia. To test the accuracy of this technique, we compared the calculated contrast-enhancement curves with those obtained by conventional manual tracing and realignment.

\section{Methods}

\subsection{Power modulation imaging}

In power modulation imaging, repeated pulses of different intensities are transmitted in the same direction. Their reflections are analysed on the basis of the hypothesis that the reflective properties of cardiac structures are mostly linear unlike those of contrast microbubbles. This technique selectively enhances microbubble-generated reflections, while those from cardiac structures and tissues are suppressed.

\subsection{Animal preparation}

Experiments were performed in 13 farm pigs (20$30 \mathrm{~kg})$. Animals were pre-treated with telazol $(2.2 \mathrm{mg} / \mathrm{kg}$, i.m.) and atropine $(0.05 \mathrm{mg} / \mathrm{kg}$, i.m. $)$ mechanically ventilated and anaesthetized with isoflurane (0.5-2.5\%). Electrocardiogram, body temperature, blood pressure and expiratory gases were monitored (Datex). Lidocaine was administered ( $1 \mathrm{mg} / \mathrm{kg}$, i.v. bolus $+4 \mathrm{mg} / \mathrm{kg} / \mathrm{hr}$ infusion) to prevent ventricular arrhythmias. An intracoronary balloon catheter (2.5-3.5 mm balloon diameter) was introduced via the right femoral artery into the LAD coronary artery under fluoroscopic guidance. The balloon was positioned near the origin of the artery to maximize the perfusion territory affected by balloon inflations.
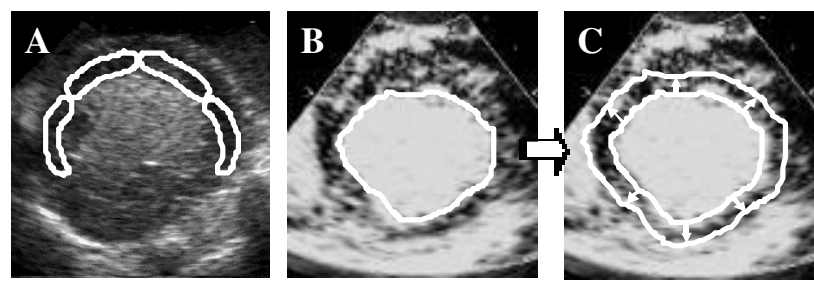

Figure 1. Traditionally, contrast regional enhancement curves are obtained by manually tracing myocardial ROIs (A). Power modulation images provide a more uniform LV cavity contrast, suitable for automated endocardial border detection (B). Expansion of the border may allow automated identification of the myocardium (C) as a basis for translation-free analysis of myocardial perfusion. 


\subsection{Imaging and protocol}

Transthoracic images were obtained using a S3 transducer (SONOS 5500, Philips). Parasternal short-axis views were obtained at the level of the papillary muscles. Power modulation was activated at mechanical indices of $0.1-0.2$, gain $65-75$, pulse repetition frequency 3.7 , and low line density. Contrast enhancement was achieved using intravenous infusion of Definity (DuPont). Infusion rates were optimised for dense opacification of the LV cavity without attenuation, and with clearly visualized intramyocardial contrast.

To track ischemic changes in myocardial perfusion, power modulation images triggered at end-systole were acquired over $60 \mathrm{sec}$, including $15 \mathrm{sec}$ control, $30 \mathrm{sec}$ complete coronary occlusion, and $15 \mathrm{sec}$ reperfusion. During each phase, a high-energy ultrasound pulse (mechanical index 1.7) was transmitted to cause contrast destruction and image subsequent replenishment. A total of 25 whole datasets (control, occlusion and reperfusion) was obtained and processed as follows.

\subsection{Image analysis}

Digital images were first cropped to contain the LV cavity and the surrounding myocardial tissue.

A border detection algorithm based on quad-tree decomposition and seeded region growing (SRG) [3] was applied to semi-automatically detect the myocardial tissue in every frame. First, the image was divided into blocks according to the condition:

$$
|\max (I)-\min (I)| \leq \text { threshold }
$$

where $I$ represents the pixel intensity. The minimum size for each block was set to $2 \times 2$ pixels and the optimal threshold value was experimentally determined as equal to 55. Then, the SRG procedure was applied in order to define the myocardial region: the image was divided in two parts (top, T, and bottom, B); for each part, a seed point was automatically located in the myocardium, as the pixel with minimum intensity level along a vertical line positioned in the middle of the image. Given the seeds, SRG determined image tessellation in two regions $\left(R_{T}\right.$ and $R_{B}$ ) by assuming that each connected component $(i, j)$ of a region meets the following homogeneity criteria:

$$
\begin{array}{ll}
\left|I\left(i_{T}, j_{T}\right)-I(i, j)\right| \leq h & \forall(i, j) \in R_{T} \\
\left|I\left(i_{B}, j_{B}\right)-I(i, j)\right| \leq h-\text { slope } \cdot i_{B} & \forall(i, j) \in R_{B}
\end{array}
$$

where $\left(i_{T}, j_{T}\right)$ and $\left(i_{B}, j_{B}\right)$ represent the coordinates of the top and bottom seed points respectively and $h$ is the threshold value. This criterion allows including in $R_{T}$ all connected pixel blocks, obtained by the quad-tree decomposition, where the intensity does not differ from the seed point intensity more then a user-defined threshold value. In the bottom half of the image, a linear depth-dependent threshold was introduced to compensate
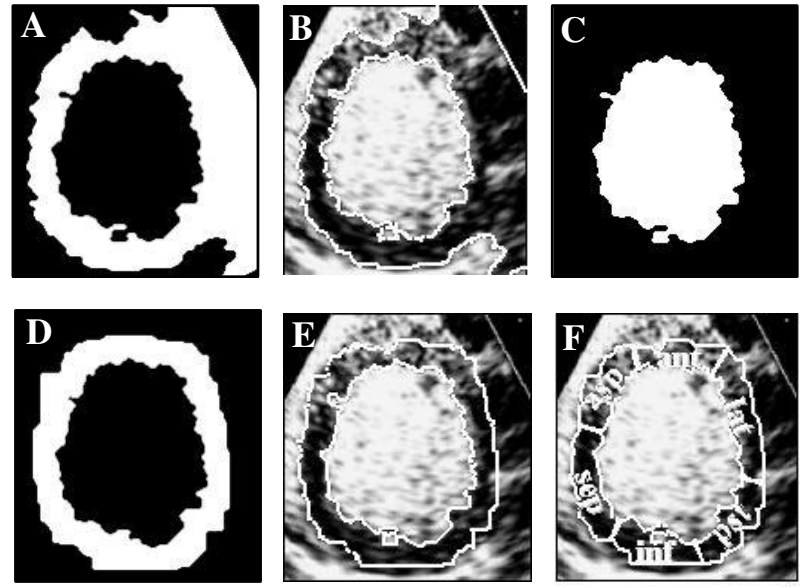

Figure 2. Automated identification of myocardial ROIs: the result of region growing (A) was used to identify the inner and outer contours (B). LV cavity was then extracted (C), and expanded to a preset width (D). Myocardium was obtained (E) as the logical AND operation between (A) and (D), and divided into ROIs (F) corresponding to coronary perfusion territories.

for signal attenuation.

Finally, the perimeter of the LV myocardial tissue was extracted as the boundary of the region $R=R_{T} \cup R_{B}$, and superimposed on the original image (Fig. 2, A-B) to allow visual verification, and adjustment of $h$ if necessary. The inner and the outer borders of the myocardial tissue represent the LV endocardial and epicardial estimated position respectively.

A region $W$ of a predefined maximum width (set to 15 pixels) was then obtained by dilating the inner contour (Fig. 2, D). The epicardial contour was used as a local constraint, preventing the inclusion in $W$ of high intensity extra-myocardial structures (Fig. 2, E). The myocardium $W$ was automatically divided into six $60^{\circ}$ segments (ant: anterior; asp: antero-septal; sep: septal; inf: inferior; pst: posterior; lat: lateral), corresponding to the ROIs used to assess perfusion (Fig. 2, F) [4]. For each segment, mean pixel intensity after high energy pulse was calculated frame-by-frame and fitted with the following exponential function of time: $\quad y=A \cdot\left(1-e^{-\beta \cdot t}\right)$ where $A, \beta$ and $A \cdot \beta$ are related to microvascular blood volume and flow [5].

\subsection{Validation with manual tracings}

10 datasets for each experimental phase were analyzed as follows. For each dataset, 5 consecutive end-systolic frames were selected during a steady-state, following contrast replenishment. For each frame, the region $W$ corresponding to the myocardial tissue was obtained both manually and automatically, and mean pixel intensity was calculated. For each dataset, SD/mean was calculated as an index of the noise level present in the series extracted with the manual and the automated techniques. 


\subsection{Statistical analysis}

The values of the parameters $A, \beta$ and $A \cdot \beta$ were averaged for all animals during each experimental phase, including control, coronary occlusion and reperfusion. Data are displayed as mean \pm SD. Two-way analysis of variance with repeated measures was used to test significance of differences between phases for all segments. Differences were considered significant when p-value below 0.05 was obtained for a segment, compared to control conditions. In addition, to emphasize ischemia- and reperfusion-induced changes despite possible inter-segmental baseline differences, perfusion and function indices were normalized by their control values, averaged and displayed. Computed SD/mean indices relevant to the automated and manual techniques were compared using paired t-test.

\section{Results}

High energy ultrasound pulses caused transient destruction of the reflective properties of the microbubbles, which were then gradually restored to baseline level. Reversible perfusion defects caused by coronary occlusion were visualized in real time: balloon inflation resulted in a gradual decrease in intramyocardial contrast, reflecting the regional lack of microbubble inflow in the LAD territory. Balloon deflation resulted in a rapid increase in the intramyocardial contrast, reflecting the hyperemic effects of reperfusion.

The SRG algorithm was able to detect myocardial contours in all the 952 examined frames, with a mean computation time of $17 \mathrm{sec} / \mathrm{frame}$.

Figure 3 shows an example of the replenishment curves obtained in all segments: under control conditions, during ischemia and reperfusion. Although differences between segments were noted, including reduced regional intensity in posterior and lateral segments due to contrast
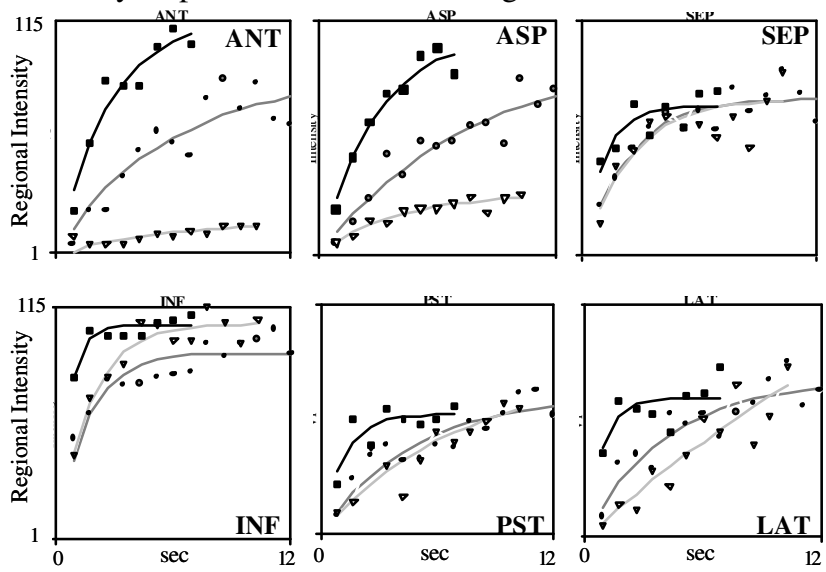

Figure 3. Example of contrast replenishment curves obtained under control conditions (circles), during coronary occlusion (triangles) and reperfusion (squares). Note the ischemia-induced changes in the anterior and antero-septal attenuation, ischemia-induced changes were evident in the LAD perfusion territory (mostly in the anterior and anteroseptal segments), followed by an increase above baseline levels as a result of reperfusion hyperemia.

In all animals, reversible perfusion defects in the LAD territory were quantitatively confirmed by the changes in replenishment curve parameters (Fig. 4). At baseline, the parameters $A$ and $A * \beta$ were similar in the ischemic and non-ischemic segments (A: $71 \pm 28$ intensity units, i.u.; $A * \beta: 37 \pm 20$ i.u. $* \mathrm{sec}^{-1}$ ). During occlusion, $A$ and $A * \beta$ decreased significantly in the anterior and anteroseptal segments (A: $28 \pm 15$ i.u.; $A * \beta: 10 \pm 8$ i.u. $* \sec ^{-1}$ ), but remained unchanged in the non-ischemic segments. During reperfusion, the values of $A$ and $A * \beta$ increased above the control level in the ischemic segments (A:112 \pm 40 i.u.; $A * \beta: 51 \pm 32$ i.u. $* \mathrm{sec}^{-1}$ ), but remained essentially unchanged in the other segments. Although no significant changes were noted with occlusion in $\beta$, this index increased in ischemic segments during reperfusion $\left(0.54 \pm 0.26 \mathrm{sec}^{-1}\right.$ compared to baseline $\left.0.37 \pm 0.18 \mathrm{sec}^{-1}\right)$.

The comparison between measured contrast levels obtained from the automatically and manually defined ROIs showed high linear correlation $\left(r^{2}=0.90\right)$. Analysis of data obtained during steady-state segments showed a significant decrease in $\mathrm{SD} /$ mean with the automated $(0.08 \pm 0.037)$ versus manual $(0.12 \pm 0.04)$ techniques.
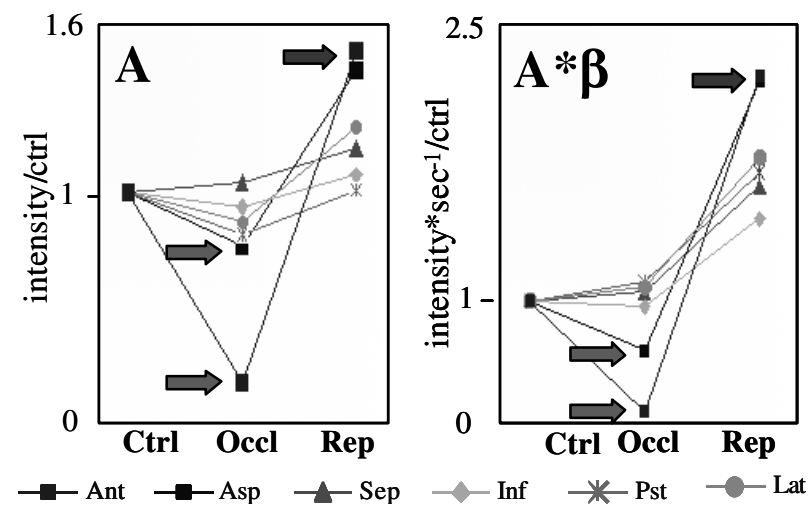

Figure 4. Summary of control-normalized indices of regional myocardial perfusion measured in 25 pigs. As a result of coronary occlusion, both $\mathrm{A}$ and $\mathrm{A} * \mathrm{~B}$ decreased in anterior and antero-septal segments, whereas other regions showed no significant change vs control ( $\mathrm{p}<0.05)$. During reperfusion, both indices increased in ischemic segments to levels that were higher than control values.

\section{Discussion}

Although multiple methods based on analysis of contrast-enhanced echocardiographic images to quantify myocardial perfusion have been described in the past decade [6-10], no automated procedure is currently 
available to identify and track myocardial tissue. This is done manually by tracing and frame-by-frame realignment of myocardial regions of interest. We previously demonstrated that power modulation imaging, when used with low-energy ultrasound, can provide uniform LV cavity opacification suitable for automated endocardial border detection [1,2]. In this study, this concept was extended to automated detection of myocardial tissue boundaries, as a tool to address the issue of frame-by-frame cardiac translation and deformation, which is essential for accurate analysis of myocardial perfusion from sequences of contrastenhanced echocardiographic images.

The seeded region growing procedure, based on the similarity of pixels within homogeneous regions, allowed the detection of the endocardial and epicardial contours. Knowing the position of these contours on a frame-byframe basis allows the quantification of the mean pixel intensity in the area between them over time. To circumvent the incomplete epicardial visualization, the endocardial border was expanded to define a fixed-width myocardial band using the detected epicardium as a local constraint. This procedure, combined with the automated myocardial segmentation, obviated the subjectivity intrinsic to manual tracing of ROIs, and improved the signal-to-noise ratios of the contrast enhancement curves.

Data obtained in animal experiments, during acute ischemia and reperfusion, demonstrated the feasibility of applying this approach to transthoracic contrast-enhanced echocardiographic images. Moreover, this technique was shown to be sensitive enough to detect acute, reversible changes in myocardial perfusion caused by short-term coronary occlusions. In addition to control data obtained from ischemic segments at baseline, perfusion territories of other coronary arteries provided a simultaneously obtained reference for comparison.

\section{Conclusions}

This is the first study to attempt automated identification of myocardial regions of interest geared toward objective, translation-free analysis of myocardial contrast enhancement from echocardiographic images.

Our hypothesis that power modulation imaging with low mechanical indices provides a basis for automated endocardial border detection allowed us to measure intramyocardial contrast enhancement over time without manual tracing and realignment of myocardial regions of interest. This novel automated technique was successfully applied to data obtained in an animal model of acute ischemia and allowed us to quantify the effects of coronary occlusion and reperfusion. The results of this study indicate that this approach may provide a useful tool for fast and objective assessment of myocardial perfusion and thus contribute towards the improved accuracy of echocardiographic diagnosis of ischemic heart disease.

\section{References}

[1] Mor-Avi V, Caiani EG, Collins KA, Korcarz CE, Bednarz JE, Lang RM. Combined assessment of myocardial perfusion and regional left ventricular function by analysis of contrast-enhanced power modulation images. Circulation 2001;104(3):352-7.

[2] Caiani EG, Lang RM, DeCara J, Bednarz JE, Weinert L, Korcarz CE, Collins KA, Mor-Avi V. Objective assessment of left ventricular wall motion from contrast-enhanced power modulation images. J Am Soc Echocardiogr 2002:15(2):118-28.

[3] Adams R, Bischof L. Seeded region growing. IEEE Trans on Pattern Analysis and Machine Intelligence 1994;16:641-7.

[4] Pandian N, Kieso R, Kerber R. Two-dimensional echocardiography in experimental coronary stenosis. Circulation 1982;98:1912-20.

[5] Wei K, Jayaweera AR, Firoozan S, Linka A, Skyba DM, Kaul S. Quantification of myocardial blood flow with ultrasound-induced destruction of microbubbles administered as a constant venous infusion. Circulation 1998;97:473-83.

[6] Vandenberg BF, Kieso R, Fox-Eastham K, Chilian W, Kerber RE. Quantitation of myocardial perfusion by contrast echocardiography: analysis of contrast gray level appearance variables and intracyclic variability. J Am Coll Cardiol 1989;13:200-6.

[7] Mor-Avi V, David D, Akselrod S, Bitton Y, Choshniak I. Myocardial regional blood flow: quantitative measurement by computer analysis of contrast enhanced echocardiographic images. Ultrasound Med Biol 1993;19:619-33.

[8] Skyba DM, Jayaweera AR, Goodman NC, Ismail S, Camarano G, Kaul S. Quantification of myocardial perfusion with myocardial contrast echocardiography during left atrial injection of contrast. Circulation 1994;90:1513-21.

[9] Masugata H, Peters B, Lafitte S, Strachan GM, Ohmori K, DeMaria AN. Quantitative assessment of myocardial perfusion during graded coronary stenosis by real-time myocardial contrast echo refilling curves. J Am Coll Cardiol 2001;37:262-9.

[10] Villanueva FS, Gertz EW, Csikari M, Pulido G, Fisher D, Sklenar J. Detection of coronary artery stenosis with power Doppler imaging. Circulation 2001;103:2624-30

Address for correspondence:

Enrico G Caiani, Ph.D.

Dipartimento di Bioingegneria

Politecnico di Milano

P.zza L. da Vinci 32

20133 Milano, Italy

caiani@biomed.polimi.it 